\title{
Predictive Factors for Non-Sentinel Lymph Node Metastasis in the Case of Positive Sentinel Lymph Node Metastasis in Two or Fewer Nodes in Breast Cancer
}

\author{
Chie Toshikawa ${ }^{a}$, Yu Koyama ${ }^{a}$, b, d, Masayuki Nagahashia, Kumiko Tatsuda ${ }^{a}$, Kazuki Moroa, Junko Tsuchida ${ }^{a}$, \\ Miki Hasegawa ${ }^{a}$, Toshiyuki Niwano ${ }^{a}$, Naoko Manba ${ }^{a}$, Mayuko Ikarashia, Hitoshi Kameyama ${ }^{a}$, \\ Takashi Kobayashia, Shin-ichi Kosugia, c, Toshifumi Wakai ${ }^{\mathrm{a}}$
}

\begin{abstract}
Background: In breast cancer, recent clinical trials have shown that sentinel lymph node biopsy (SLNB) alone without axillary lymph node dissection results in excellent prognosis if there is sentinel lymph node (SLN) metastasis in two or fewer nodes. The aim of the present study was to investigate the association between non-SLN metastasis and clinicopathological factors in case of SLN metastasis in two or fewer nodes in breast cancer.
\end{abstract}

Methods: Patients who underwent SLNB for invasive breast cancer and were found to have positive SLN in two or fewer nodes were evaluated. The associations between non-SLN metastasis and clinicopahological factors were examined. Statistical analyses were performed using the Mann-Whitney and Chi-square tests, with statistical significance set at $\mathrm{P}<0.05$.

Results: A total of 358 patients were enrolled during the study period and all of these patients were female and 54 patients had SLN metastasis (15\%). Positive SLN in two or fewer nodes was identified in 44 patients $(81.5 \%)$. Among these patients, 17 (38.6\%) were found to have non-SLN metastasis. Non-SLN metastasis was associated with invasive tumor size $(\mathrm{P}=0.015)$ and lymphatic involvement $(\mathrm{P}=$ 0.035). Multivariate analysis showed that tumor size $(\mathrm{P}=0.011)$ and lymphatic involvement $(\mathrm{P}=0.019)$ remained significant independent predictors of non-SLN metastasis, and that an invasive tumor size cut-off point of $28 \mathrm{~mm}$ was useful for dividing patients with positive SLN in two or fewer nodes into non-SLN-positive and non-SLNnegative groups.

Manuscript accepted for publication May 08, 2015

aDivision of Digestive \& General Surgery, Niigata University Graduate School of Medical \& Dental Sciences, 1-757 Asahimachi, Niigata, Niigata 951-8510, Japan

${ }^{b}$ Department of Nursing, Niigata University Graduate School of Health Sciences, 2-746 Asahimachi, Niigata, Niigata 951-8518, Japan

'Department of Digestive and General Surgery, Uonuma Institute of Community Medicine, Niigata University Medical and Dental Hospital, 4132 Urasa, Minami-Uonuma, Niigata 949-7302, Japan

${ }^{\mathrm{d} C}$ Corresponding Author: Yu Koyama, Department of Nursing, Niigata University Graduate School of Health Sciences, 2-746 Asahimachi, Niigata, Niigata 951-8518, Japan. Email: yukmy@clg.niigata-u.ac.jp

doi: http://dx.doi.org/10.14740/jocmr2195w
Conclusions: Non-SLN metastasis was found in more than $30 \%$ of patients with SLN metastasis present in two or fewer nodes. Large tumor size and the presence of lymphatic involvement were significantly associated with non-SLN metastasis.

Keywords: Breast cancer; Sentinel lymph node; Non-sentinel lymph node; Metastasis; Lymphatic involvement; Invasive tumor size

\section{Introduction}

Non-sentinel lymph node (SLN) metastasis has been an important issue for planning further treatment of node positive breast cancer, because axillary lymph node metastasis has been a strong prognostic indicator for the patients with invasive breast cancer while new tumor markers have been widely studied [1-3]. Axillary lymph node dissection (ALND) is the standard management approach for preoperatively diagnosed nodepositive breast cancer [4]. Since the 1990s, the introduction of sentinel lymph node biopsy (SLNB) has resulted in changes in the management of the axilla [5]. SLNB for clinically N0 breast cancer and ALND for positive SLN have become the standard procedures.

Recent clinical trials suggest that there is no difference in outcome between patients with positive SLN if they are treated with ALND or given no further axillary surgery $[6,7]$. These studies raise doubts concerning the role of SLNB. A new trial compared SLNB with the assessment of whether an axillary ultrasound is negative in patients with small breast cancer [8]. SLN metastasis is observed in approximately $30 \%$ of SLNBs [9], so it is important to predict the axillary node status before SLNB. Various clinicopathological factors have been identified as independent predictors of axillary lymph node metastasis in early stage breast cancer [10]. These factors include clinical palpability [11-14], tumor size [11-17], lymphatic or vascular involvement [11-15, 17], tumor grade [11, 14], hormone receptor (HR) status $[16,17]$, age $[12,15,16]$, and molecular subtype classification $[3,10,18-26]$. Predicting the non-SLN status is important because both the American College of Surgeons Oncology Group (ACOSOG) Z0011 trial [6] and the International Breast Cancer Study Group Trial 23-01 (IBCSG 23-01) [7] indicated that ALND should be avoided if SLN metastases are detected in only one or two nodes. Analyt- 
Table 1. Association Between Non-Sentinel Lymph Node Metastasis and Clinicopathological Features $(n=44)$

\begin{tabular}{|c|c|c|c|c|}
\hline \multirow{2}{*}{ Characteristics } & \multirow{2}{*}{ No. of patients } & \multicolumn{2}{|c|}{ Non-SLN metastasis } & \multirow{2}{*}{ P value } \\
\hline & & Negative (\%) & Positive (\%) & \\
\hline Age (years) & & & & $>0.999$ \\
\hline$\leq 50$ & 25 & $15(60.0)$ & $10(40.0)$ & \\
\hline$>50$ & 19 & $12(63.2)$ & $7(36.8)$ & \\
\hline Invasive tumor size (mm) & & & & 0.062 \\
\hline$\leq 20$ & 22 & $17(77.3)$ & $5(22.7)$ & \\
\hline$>20$ & 22 & $10(45.5)$ & $12(54.5)$ & \\
\hline Histological grade & & & & 0.108 \\
\hline I & 28 & $20(71.4)$ & $8(28.6)$ & \\
\hline II-III & 16 & $7(43.8)$ & $9(56.2)$ & \\
\hline Lymphatic involvement & & & & 0.035 \\
\hline Negative & 32 & $23(71.9)$ & $9(28.1)$ & \\
\hline Positive & 12 & $4(33.3)$ & $8(66.7)$ & \\
\hline Venous involvement & & & & $>0.999$ \\
\hline Negative & 37 & $23(62.2)$ & $14(37.8)$ & \\
\hline Positive & 7 & $4(57.1)$ & $3(42.9)$ & \\
\hline ER and/or PR & & & & $>0.999$ \\
\hline Negative & 3 & $2(66.7)$ & $1(33.3)$ & \\
\hline Positive & 41 & $25(61.0)$ & $16(39.0)$ & \\
\hline HER2 & & & & $>0.999$ \\
\hline Negative & 36 & $22(61.1)$ & $14(38.9)$ & \\
\hline Positive & 8 & $5(62.5)$ & $3(37.5)$ & \\
\hline Molecular subtype & & & & 0.867 \\
\hline Luminal A & 33 & $20(60.6)$ & $13(39.4)$ & \\
\hline Luminal B & 7 & $4(57.1)$ & $3(42.9)$ & \\
\hline HER2 & 1 & $1(100)$ & $0(0)$ & \\
\hline Triple negative & 3 & $2(66.7)$ & $1(33.3)$ & \\
\hline Ki-67 labeling index & & & & 0.359 \\
\hline$<14 \%$ & 25 & $17(68.0)$ & $8(32.0)$ & \\
\hline$\geq 14 \%$ & 19 & $10(52.6)$ & $9(47.4)$ & \\
\hline
\end{tabular}

SLN: sentinel lymph node; ER: estrogen receptor; PR: progesterone receptor.

ical tools have been developed to predict the risk of non-SLN metastasis if positive SLNs are found [27-33], but these tools yield a false negative rate of 7-41\% (ALND for $<10 \%$ risk of non-SLN metastasis) [34]. Recently, we reported that SLN metastasis was associated with younger age, large tumor size and prominent lymphovascular involvement; however, nonSLN metastasis was hard to predict using clinicopathological factors [35].

The aim of the present study was to investigate the association between non-SLN metastasis and clinicopathological factors, particularly in the case of SLN metastasis in two or fewer nodes, in breast cancer.

\section{Materials and Methods}

\section{Patient selection}

Patients with invasive breast cancer who underwent SLNB at Niigata University Hospital between January 2010 and December 2014 were eligible for inclusion in the study, which was a retrospective chart review. ALND was performed in patients with macro- and micrometastasis in SLN; however, ALND was avoided in patients with isolated tumor cells in SLN. Of the patients who were positive for SLN metastasis, those with 
SLN metastases in two or fewer nodes were evaluated further. Only those patients with complete data for clinicopathological factors (age, clinical and pathological tumor size, HR and human epidermal growth factor receptor 2 (HER2) status, and Ki-67 labeling index) were enrolled in the study $(n=44)$. The data for these patients were analyzed following approval from the Institutional Review Board.

\section{Pathological assessment}

Immunohistochemical (IHC) estrogen receptor (ER) and progesterone receptor (PR) status was assessed and tumors were deemed positive for each receptor if at least $10 \%$ of the invasive tumor cells in a section exhibited nuclear staining for that particular receptor. HER2 expression was examined by IHC. A gene amplification assay using fluorescence in situ hybridization (FISH) was used in cases when it was difficult to decide the HER2 status by IHC. Ki-67 was also examined by $\mathrm{IHC}$, and the results are expressed as the percentage of tumor cells stained by the antibody, as described previously [36]. Hematoxylin-eosin staining was used to assess lymphatic and vascular involvement, as well as histologic grading, which was defined according to the Scarff-BloomRichardson system [37]. SLN metastasis was assessed by intraoperative examination of frozen sections, as well as being re-evaluated postoperatively using fixed sections. Breast cancer staging was according to the TNM classification as proposed by the American Joint Committee on Cancer (AJCC). All IHC evaluations were performed by several well-trained pathologists.

Patients were assigned into four subgroups, as proposed in the St Gallen International Expert Consensus [38] based on the results of their ER, PR, and HER2 status and Ki-67 leveling index [39], as follows: 1) a luminal A group that was ER or PR positive, HER2 negative, and had a Ki-67 labeling index $<$ $14 \%$; 2) a luminal B group that was ER or PR positive, HER2 positive, or had a Ki-67 labeling index $(\geq 14 \%$; 3) an HER2 group that was ER and PR negative and HER2 positive; and 4) a triple negative group that was negative for ER, PR, and HER2.

\section{Statistical analysis}

The associations between non-SLN metastasis and clinicopahological factors (age, invasive tumor size, nulcear grade, lymphatic or venous involvement, ER and/or PR status, HER2 status, molecular subtypes, and Ki-67 labeling index) were examined. Statistical analyses were performed using MannWhitney's U-test and the Chi-squared test, and multivariate analysis was performed using the logistic regression model. The diagnostic accuracy of invasive tumor size was assessed by receiver operating characteristic (ROC) analysis. The area under the ROC curve (AUC) was used to measure model discrimination. The AUC can range from 0.5 (which indicates a test with no information) to 1.0 (which indicates a perfect test). Statistical significance was set at $\mathrm{P}<0.05$.

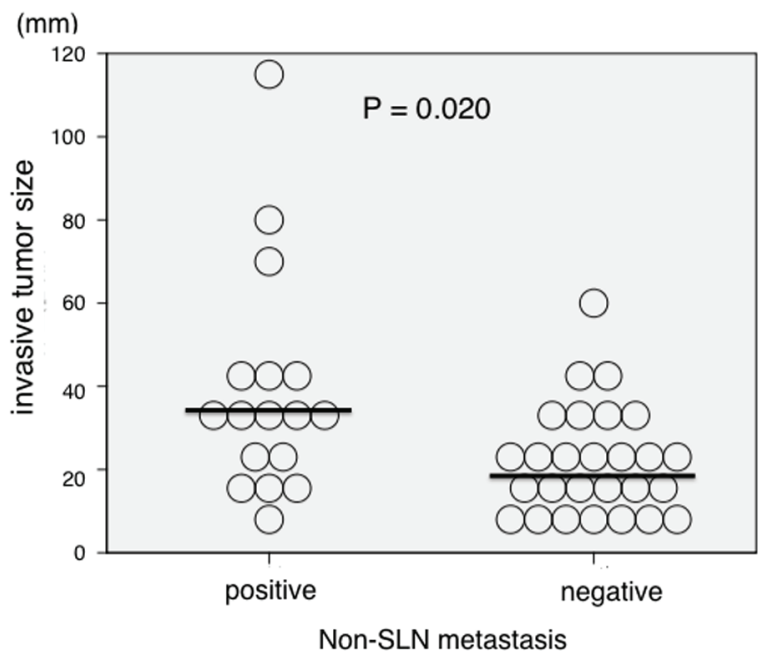

Figure 1. Association between invasive tumor size and non-sentinel lymph node $(S L N)$ metastasis $(n=44)$. Invasive tumor size was significantly larger in patients who were positive for non-SLN metastasis compared with those who were negative (median size 35.0 vs. $20 \mathrm{~mm}$, respectively; $\mathrm{P}=0.020$ ).

\section{Results}

\section{SLN and non-SLN metastasis}

A total of 358 patients were enrolled during the study period. All patients were female and 54 had SLN metastasis (15\%). Of the patients who were positive for SLN metastases, positive SLN in two or fewer nodes were obseved in 44 patients (81.5\%). Among these 44 patients, non-SLN metastasis was observed in 17 patients $(38.6 \%)$. Thirty-four patients $(72.3 \%)$ had positive SLN in one node and 10 patients $(22.7 \%)$ had positive SLN in two nodes.

\section{Patient characteristics and clinicopathological factors}

Mean patient age was 51.6 years; however, there was no association between age and non-SLN metastasis (Table 1). Univariate analysis revealed that non-SLN metastasis was significantly associated with lymphatic involvement $(\mathrm{P}=0.035)$; however, there was no significant association between nonSLN metastasis and invasive tumor size, nulcear grade, venous involvement, ER and/or PR status, HER2 status, molecular subtype, or Ki-67 labeling index.

\section{Invasive tumor size and non-SL metastasis}

Conversely, median invasive tumor size was significantly larger in patients who were positive for non-SLN metastasis compared with metastasis negative patients $(35.0$ vs. $20 \mathrm{~mm}$, respectively; $\mathrm{P}=0.020$; Fig. 1). However, there was no significant association between non-SLN metastasis and invasive 


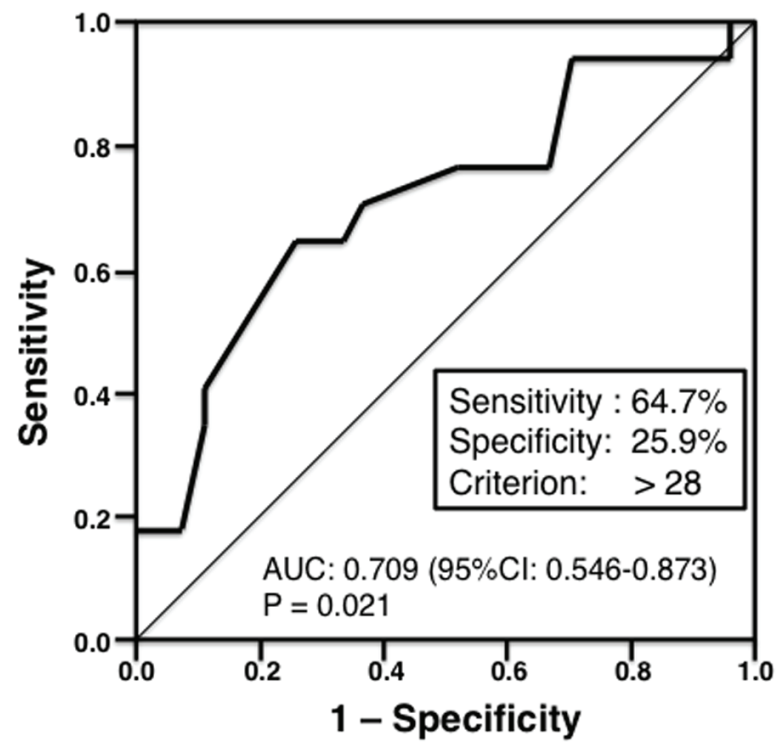

Figure 2. Receiver operating characteristic (ROC) analysis of invasive tumor size $(n=44)$. The ROC analysis identified a cut-off point of 28 $\mathrm{mm}$ (area under the curve 0.709 ; sensitivity $64.7 \%$; specificity $25.9 \%$; $P=0.021)$.

tumor size if the cut-off point was $20 \mathrm{~mm}$. Therefore, we invastigated the threshold value for invasive tumor size that differentiated patients with non-SLN metastasis using ROC analysis. ROC analysis identified a cut-off point of $28 \mathrm{~mm}$ (AUC 0.709; sensitivity 64.7\%; specificity $25.9 \%$; $\mathrm{P}=0.021$; Fig. 2). We then used this cut-off point of $28 \mathrm{~mm}$ to reassess patients. As indicated in Table 2, using a cut-off value of $28 \mathrm{~mm}$ resulted in a significant association between invasive tumor size and non-SLN metastasis $(\mathrm{P}=0.015)$.

\section{Multivariate analysis of predictor for non-SLN metastasis}

Multivariate analysis showed that both lymphatic involvement $(\mathrm{P}=0.019$; relative risk (RR): $7.255 ; 95 \%$ confidence interval (CI): 1.388 - 37.929) and invasive tumor size $(\mathrm{P}=0.011$; $\mathrm{RR}$ : 7.083; 95\% CI: 1.560 - 32.153) were significant factors predicting non-SLN metastasis (Table 3).
Table 3. Multivariate Analysis of Clinicopathological Factors $(\mathrm{n}=44)$

\begin{tabular}{|lll}
\hline Variable & RR $(95 \%$ CI $)$ & P value \\
\hline $\begin{array}{l}\text { Lymphatic involvement } \\
\quad \text { Negative }\end{array}$ & 1.000 & \\
$\quad$ Positive & $7.255(1.388-37.929)$ & 0.019 \\
Invasive tumor size & & \\
$\quad \leq 28 \mathrm{~mm}$ & 1.000 & \\
$>28 \mathrm{~mm}$ & $7.083(1.560-32.153)$ & 0.011 \\
\hline
\end{tabular}

$\mathrm{RR}$ : relative risk; $\mathrm{Cl}$ : confidence interval.

\section{Discussion}

SLNB for clinically node-negative breast cancer has become a standard procedure worldwide and it is important to determine pathologically whether the node is negative before surgery. Although ALND has been the standard procedure if patients are positive for SLN metastasis, recent clinical trials suggest that ALND is unnecessary if positive SLN metastasis is detected in one or two nodes. The ACOSOG Z0011 study [6] showed that SLNB alone without ALND results in extremely low locoregional recurrence and excellent overall survival comparable to that in patients undergoing ALND if SLN metastasis is present in two or fewer nodes. In the present study, we investigated patients with SLN metastasis in two or fewer nodes to elucidate predictive clinicopathological factors in the case of SLN metastasis in two or fewer nodes. The results revealed a $>30 \%$ risk for non-SLN metastasis even if SLN metastasis occurred in one or two nodes only. Breast surgeons must understand the risk of renmant non-SLN metastasis if they do not perform additional ALND for patients with SLN metastasis in two or fewer nodes. Thus, predictions of non-SLN metastasis in the case of positive SLN metastasis in two or fewer nodes are necessary when making decisions regarding additional ALND.

Previous studies have reported that younger age, higher pT stage, or lymphovascular involvement are independent predictors of SLN metastasis [11-17], with HR and histological grade good predictors of positive SLN [11, 14, 16, 17]. With regard to the prediction of non-SLN metastases, younger age, large tumor size or higher pT stage, lymphovascular involve-

Table 2. Non-Sentinel Lymph Node Metastasis and Cut-Off Points of Invasive Tumor Size $(n=44)$

\begin{tabular}{|c|c|c|c|c|}
\hline \multirow{2}{*}{ Invasive tumor size } & \multirow{2}{*}{ No. of patients } & \multicolumn{2}{|c|}{ Non-SLN metastasis } & \multirow{2}{*}{ P value } \\
\hline & & Negative (\%) & Positive (\%) & \\
\hline Cut-off point $20 \mathrm{~mm}$ & & & & 0.062 \\
\hline$\leq 20 \mathrm{~mm}$ & 22 & $17(77.3)$ & $5(22.7)$ & \\
\hline$>20 \mathrm{~mm}$ & 22 & $10(45.5)$ & $12(54.5)$ & \\
\hline Cut-off point $28 \mathrm{~mm}$ & & & & 0.015 \\
\hline$\leq 28 \mathrm{~mm}$ & 26 & $20(76.9)$ & $6(23.1)$ & \\
\hline$>28 \mathrm{~mm}$ & 18 & $7(38.9)$ & $11(61.1)$ & \\
\hline
\end{tabular}

SLN: sentinel lymph node. 
ment, extracapsular invasion, the ratio of positive SLNs to the total number of harvested SLNs, or total tumoral load in the SLNs assessed by one-step nucleic acid amplification have been reported as useful markers [40-44]. However, these studies were not performed in patients with positive SLN metastasis in two or fewer nodes. Therefore, in the present study, we investigated the association between non-SLN metastasis and clinicopathological factors, particularly in the case of SLN metastasis in two or fewer nodes, in breast cancer. The findings show that invasive tumor size and lymphatic involvement are significantly associated with non-SLN metastasis in the case of SLN metastasis in two or fewer nodes. We investigated the association between non-SLN metastasis and invasive tumor size initially using a cut-off point of $20 \mathrm{~mm}$ because $20 \mathrm{~mm}$ is the point dividing T1 and T2; however, there was no significant association between non-SLN and invasive tumor size using $20 \mathrm{~mm}$ as the cut-off point. However, invasive tumor size was significantly larger in patients who were positive for non-SLN metastasis compared with those who were metastasis negative (Fig. 1). So, we invastigated the threshold value of invasive tumor size that differentiated patients with non-SLN metastasis using ROC analysis. The results of ROC analysis identified 28 $\mathrm{mm}$ as the most useful cut-off point to discriminate between non-SLN metastasis positive and negative patients.

The main limitations of the present study include the retrospective nature of the analysis and the small number of patients who have had positive SLN metastasis at two or fewer nodes. We believe, however, that these limitations did not greatly affect the results of the study as the differences between nonSLN positive and negative were too marked to have resulted from bias. Our results thus provide useful information on the risk factors for renmant non-SLN metastasis under the condition of SLN metastasis at two or fewer nodes.

Finally, both lymphatic involvement and invasive tumor size (cut-off point $28 \mathrm{~mm}$ ) were independent predictors of non-SLN metastasis in the case of SLN metastasis in two or fewer nodes. Systemic therapies, including hormone therapy, cytotoxic chemotherapy, and/or molecular targeting drugs and/ or radiation therapy, remain extremely important, particularly in the treatment of node-positive breast cancer. Therefore, the results of the present study will contribute to decision-making with regard to the addition of ALND, systemic therapy, and/ or radiation therapy in the case of SLN metastasis in two or fewer nodes.

\section{Conclusion}

Non-SLN metastasis was found in more than $30 \%$ of patients in the present study, even if SLN metastasis was present in two or fewer nodes, and large tumor size and the presence of lymphatic involvement were significanly associated with nonSLN metastasis.

\section{Conflicts of Interest}

The authors declare they have no conflicts of interest.

\section{References}

1. Lee JH, Kim SH, Suh YJ, Shim BY, Kim HK. Predictors of axillary lymph node metastases (ALNM) in a Korean population with T1-2 breast carcinoma: triple negative breast cancer has a high incidence of ALNM irrespective of the tumor size. Cancer Res Treat. 2010;42(1):30-36.

2. Cserni G, Bianchi S, Vezzosi V, Arisio R, Bori R, Peterse JL, Sapino A, et al. Sentinel lymph node biopsy and non-sentinel node involvement in special type breast carcinomas with a good prognosis. Eur J Cancer. 2007;43(9):1407-1414.

3. Rosen PP, Groshen S, Kinne DW, Norton L. Factors influencing prognosis in node-negative breast carcinoma: analysis of $767 \mathrm{~T} 1 \mathrm{~N} 0 \mathrm{M} 0 / \mathrm{T} 2 \mathrm{~N} 0 \mathrm{M} 0$ patients with longterm follow-up. J Clin Oncol. 1993;11(11):2090-2100.

4. Consensus statement: treatment of early-stage breast cancer. National Institutes of Health Consensus Development Panel. J Natl Cancer Inst Monogr. 1992;11:1-5.

5. Giuliano AE, Kirgan DM, Guenther JM, Morton DL. Lymphatic mapping and sentinel lymphadenectomy for breast cancer. Ann Surg. 1994;220(3):391-398; discussion 398-401.

6. Giuliano AE, Hunt KK, Ballman KV, Beitsch PD, Whitworth PW, Blumencranz PW, Leitch AM, et al. Axillary dissection vs no axillary dissection in women with invasive breast cancer and sentinel node metastasis: a randomized clinical trial. JAMA. 2011;305(6):569-575.

7. Galimberti V, Cole BF, Zurrida S, Viale G, Luini A, Veronesi $\mathrm{P}$, Baratella $\mathrm{P}$, et al. Axillary dissection versus no axillary dissection in patients with sentinel-node micrometastases (IBCSG 23-01): a phase 3 randomised controlled trial. Lancet Oncol. 2013;14(4):297-305.

8. Gentilini O, Veronesi U. Abandoning sentinel lymph node biopsy in early breast cancer? A new trial in progress at the European Institute of Oncology of Milan (SOUND: Sentinel node vs Observation after axillary UltraSouND). Breast. 2012;21(5):678-681.

9. Canavese G, Gipponi M, Catturich A, Vecchio C, Tomei D, Nicolo G, Carli F, et al. Technical issues and pathologic implications of sentinel lymph node biopsy in earlystage breast cancer patients. J Surg Oncol. 2001;77(2):8187; discussion 88.

10. Crabb SJ, Cheang MC, Leung S, Immonen T, Nielsen TO, Huntsman DD, Bajdik CD, et al. Basal breast cancer molecular subtype predicts for lower incidence of axillary lymph node metastases in primary breast cancer. Clin Breast Cancer. 2008;8(3):249-256.

11. Barth A, Craig PH, Silverstein MJ. Predictors of axillary lymph node metastases in patients with T1 breast carcinoma. Cancer. 1997;79(10):1918-1922.

12. Olivotto IA, Jackson JS, Mates D, Andersen S, Davidson W, Bryce CJ, Ragaz J. Prediction of axillary lymph node involvement of women with invasive breast carcinoma: a multivariate analysis. Cancer. 1998;83(5):948-955.

13. Silverstein MJ, Gierson ED, Waisman JR, Colburn WJ, Gamagami P. Predicting axillary node positivity in patients with invasive carcinoma of the breast by using a 
combination of $\mathrm{T}$ category and palpability. J Am Coll Surg. 1995;180(6):700-704.

14. Silverstein MJ, Skinner KA, Lomis TJ. Predicting axillary nodal positivity in 2282 patients with breast carcinoma. World J Surg. 2001;25(6):767-772.

15. Gajdos C, Tartter PI, Bleiweiss IJ. Lymphatic invasion, tumor size, and age are independent predictors of axillary lymph node metastases in women with T1 breast cancers. Ann Surg. 1999;230(5):692-696.

16. Gann PH, Colilla SA, Gapstur SM, Winchester DJ, Winchester DP. Factors associated with axillary lymph node metastasis from breast carcinoma: descriptive and predictive analyses. Cancer. 1999;86(8):1511-1519.

17. Viale G, Zurrida S, Maiorano E, Mazzarol G, Pruneri G, Paganelli G, Maisonneuve P, et al. Predicting the status of axillary sentinel lymph nodes in 4351 patients with invasive breast carcinoma treated in a single institution. Cancer. 2005;103(3):492-500.

18. Lu X, Wang ZC, Iglehart JD, Zhang X, Richardson AL. Predicting features of breast cancer with gene expression patterns. Breast Cancer Res Treat. 2008;108(2):191-201.

19. Liu H, Fan Q, Zhang Z, Li X, Yu H, Meng F. Basal-HER2 phenotype shows poorer survival than basal-like phenotype in hormone receptor-negative invasive breast cancers. Hum Pathol. 2008;39(2):167-174.

20. Van Calster B, Vanden Bempt I, Drijkoningen M, Pochet N, Cheng J, Van Huffel S, Hendrickx W, et al. Axillary lymph node status of operable breast cancers by combined steroid receptor and HER-2 status: triple positive tumours are more likely lymph node positive. Breast Cancer Res Treat. 2009;113(1):181-187.

21. Kim MJ, Ro JY, Ahn SH, Kim HH, Kim SB, Gong G. Clinicopathologic significance of the basal-like subtype of breast cancer: a comparison with hormone receptor and Her2/neu-overexpressing phenotypes. Hum Pathol. 2006;37(9):1217-1226.

22. Nguyen PL, Taghian AG, Katz MS, Niemierko A, Abi Raad RF, Boon WL, Bellon JR, et al. Breast cancer subtype approximated by estrogen receptor, progesterone receptor, and HER-2 is associated with local and distant recurrence after breast-conserving therapy. J Clin Oncol. 2008;26(14):2373-2378.

23. Voduc KD, Cheang MC, Tyldesley S, Gelmon K, Nielsen TO, Kennecke H. Breast cancer subtypes and the risk of local and regional relapse. J Clin Oncol. 2010;28(10):16841691.

24. Reyal F, Rouzier R, Depont-Hazelzet B, Bollet MA, Pierga JY, Alran S, Salmon RJ, et al. The molecular subtype classification is a determinant of sentinel node positivity in early breast carcinoma. PLoS One. 2011;6(5):e20297.

25. Buglioni S, Di Filippo F, Terrenato I, Casini B, Gallo E, Marandino F, Maini CL, et al. Quantitative molecular analysis of sentinel lymph node may be predictive of axillary node status in breast cancer classified by molecular subtypes. PLoS One. 2013;8(3):e58823.

26. Mazouni C, Rimareix F, Mathieu MC, Uzan C, Bourgier $\mathrm{C}$, Andre F, Delaloge S, et al. Outcome in breast molecular subtypes according to nodal status and surgical procedures. Am J Surg. 2013;205(6):662-667.
27. Krag DN, Anderson SJ, Julian TB, Brown AM, Harlow SP, Costantino JP, Ashikaga T, et al. Sentinel-lymph-node resection compared with conventional axillary-lymphnode dissection in clinically node-negative patients with breast cancer: overall survival findings from the NSABP B-32 randomised phase 3 trial. Lancet Oncol. 2010;11(10):927-933.

28. Van Zee KJ, Manasseh DM, Bevilacqua JL, Boolbol SK, Fey JV, Tan LK, Borgen PI, et al. A nomogram for predicting the likelihood of additional nodal metastases in breast cancer patients with a positive sentinel node biopsy. Ann Surg Oncol. 2003;10(10):1140-1151.

29. Kohrt HE, Olshen RA, Bermas HR, Goodson WH, Wood DJ, Henry S, Rouse RV, et al. New models and online calculator for predicting non-sentinel lymph node status in sentinel lymph node positive breast cancer patients. BMC Cancer. 2008;8:66.

30. Barranger E, Coutant C, Flahault A, Delpech Y, Darai E, Uzan S. An axilla scoring system to predict non-sentinel lymph node status in breast cancer patients with sentinel lymph node involvement. Breast Cancer Res Treat. 2005;91(2):113-119.

31. Pal A, Provenzano E, Duffy SW, Pinder SE, Purushotham AD. A model for predicting non-sentinel lymph node metastatic disease when the sentinel lymph node is positive. Br J Surg. 2008;95(3):302-309.

32. Degnim AC, Reynolds C, Pantvaidya G, Zakaria S, Hoskin T, Barnes S, Roberts MV, et al. Nonsentinel node metastasis in breast cancer patients: assessment of an existing and a new predictive nomogram. Am J Surg. 2005;190(4):543-550.

33. Katz A, Smith BL, Golshan M, Niemierko A, Kobayashi W, Raad RA, Kelada A, et al. Nomogram for the prediction of having four or more involved nodes for sentinel lymph node-positive breast cancer. J Clin Oncol. 2008;26(13):2093-2098.

34. Berrang TS, Lesperance M, Truong PT, Walter C, Hayashi AH, Olivotto IA. Which prediction models best identify additional axillary disease after a positive sentinel node biopsy for breast cancer? Breast Cancer Res Treat. 2012;133(2):695-702.

35. Hasegawa M, Koyama Y, Sakata J, Tatsuda K, Sakata E, Chie Toshikawa C, Manba N, et al. Non-sentinel lymph node metastasis is hard to predict by clinicopathological factors if SLN metastasis in two or fewer nodes in breast cancer. J Curr Surg. 2014;4(1):10-16.

36. Jones RL, Salter J, A'Hern R, Nerurkar A, Parton M, ReisFilho JS, Smith IE, et al. Relationship between oestrogen receptor status and proliferation in predicting response and long-term outcome to neoadjuvant chemotherapy for breast cancer. Breast Cancer Res Treat. 2010;119(2):315323.

37. Contesso G, Mouriesse H, Friedman S, Genin J, Sarrazin $\mathrm{D}$, Rouesse J. The importance of histologic grade in longterm prognosis of breast cancer: a study of 1,010 patients, uniformly treated at the Institut Gustave-Roussy. J Clin Oncol. 1987;5(9):1378-1386.

38. Goldhirsch A, Wood WC, Coates AS, Gelber RD, Thurlimann B, Senn HJ. Strategies for subtypes--dealing with 
the diversity of breast cancer: highlights of the St. Gallen International Expert Consensus on the Primary Therapy of Early Breast Cancer 2011. Ann Oncol. 2011;22(8):17361747.

39. Guiu S, Michiels S, Andre F, Cortes J, Denkert C, Di Leo A, Hennessy BT, et al. Molecular subclasses of breast cancer: how do we define them? The IMPAKT 2012 Working Group Statement. Ann Oncol. 2012;23(12):2997-3006.

40. Meattini I, Saieva C, Bertocci S, Francolini G, Zei G, De Luca Cardillo C, Scotti V, et al. Predictive factors for additional non-sentinel lymph node involvement in breast cancer patients with one positive sentinel node. Tumori. 2015;101(1):78-83.

41. Eldweny H, Alkhaldy K, Alsaleh N, Abdulsamad M, Abbas A, Hamad A, Mounib S, et al. Predictors of non-sentinel lymph node metastasis in breast cancer patients with positive sentinel lymph node (Pilot study). J Egypt Natl
Canc Inst. 2012;24(1):23-30.

42. Moosavi SA, Abdirad A, Omranipour R, Hadji M, Razavi AE, Najafi M. Clinicopathologic features predicting involvement of non- sentinel axillary lymph nodes in Iranian women with breast cancer. Asian Pac J Cancer Prev. 2014;15(17):7049-7054.

43. Rubio IT, Espinosa-Bravo M, Rodrigo M, Amparo Viguri Diaz M, Hardisson D, Sagasta A, Duenas B, et al. Nomogram including the total tumoral load in the sentinel nodes assessed by one-step nucleic acid amplification as a new factor for predicting nonsentinel lymph node metastasis in breast cancer patients. Breast Cancer Res Treat. 2014;147(2):371-380.

44. Illyes I, Tokes AM, Kovacs A, Szasz AM, Molnar BA, Molnar IA, Kaszas I, et al. In breast cancer patients sentinel lymph node metastasis characteristics predict further axillary involvement. Virchows Arch. 2014;465(1):15-24. 